\title{
Uncertainty Estimation by Convolution Using Spatial Statistics
}

\author{
Luis Miguel Sanchez-Brea and Eusebio Bernabeu
}

\begin{abstract}
Kriging has proven to be a useful tool in image processing since it behaves, under regular sampling, as a convolution. Convolution kernels obtained with kriging allow noise filtering and include the effects of the random fluctuations of the experimental data and the resolution of the measuring devices. The uncertainty at each location of the image can also be determined using kriging. However, this procedure is slow since, currently, only matrix methods are available. In this work, we compare the way kriging performs the uncertainty estimation with the standard statistical technique for magnitudes without spatial dependence. As a result, we propose a much faster technique, based on the variogram, to determine the uncertainty using a convolutional procedure. We check the validity of this approach by applying it to one-dimensional images obtained in diffractometry and two-dimensional images obtained by shadow moire.
\end{abstract}

Index Terms-2-INTR interpolation and spatial transformations, 2-LFLT linear filtering and enhancement, 2-NOIS noise modeling, 3-OPTI optical imaging.

\section{INTRODUCTION}

I MAGES captured with charge-coupled device (CCD) cameras are sampled, so that an interpolation is required to reconstruct the continuous image from the discrete data. In most cases photosensors are placed at fixed intervals and then interpolation can be easily performed using convolution kernels. A milestone for the interpolation of regularly sampled signals is the Whittakers-Kotel'nikov-Shannon method (WKS) [1], being still a subject of research [2]-[5]. As in any other measurement process, images acquired with a CCD camera present noise [6]-[8], and the sinc kernel does not work properly. Convolution kernels that allow noise filtering have been proposed [9]-[12], but most of these interpolation methods do not calculate the uncertainty of the estimation. This can be a problem when the image is used for metrological purposes, since its knowledge is required to determine the uncertainty of the parameters measured from the image.

The standard technique for determining the uncertainty of a quantity $Q$ is to perform a number $N$ of measurements under the same conditions $\left\{Q_{i}, i=1, \ldots, N\right\}$. The most accurate estimation of $Q$ is the arithmetic mean $(\bar{Q})$, and the uncertainty of the estimation is given by [13], [14]

$$
\sigma(\bar{Q})=\left[I^{2}+\frac{s^{2}(Q)}{N}\right]^{1 / 2}
$$

Manuscript received April 19, 2005; revised February 9, 2006. The work of L. M. Sanchez-Brea was supported by the Ministerio de Educación y Ciencia of Spain, within the "Ramón y Cajal" program. The associate editor coordinating the review of this manuscript and approving it for publication was Dr. Til Aach.

The authors are with the Optics Department, Universidad Complutense de Madrid, 28040 Madrid, Spain (e-mail: sanchezbrea@fis.ucm.es).

Digital Object Identifier 10.1109/TIP.2006.877505 where $s^{2}(Q)$ is the experimental variance and $I$ is the resolution of the measuring device, that is, the smallest increment in the value of the measurand that results in a detectable increment in the output [15]. In image processing, this procedure means that several images should be acquired in order to reduce noise and compute the uncertainty.

Convolution kernels use a different approach since the noise within one single image is filtered using a convolution with the kernel. Let $Z_{i}$ be the value of the data at each pixel of the image. Pixels are uniformly placed at $\mathbf{x}_{i} \subset \mathbb{R}^{D}$ (being $D$ the dimension) with a sampling distance at each dimension $\Delta_{1}, \ldots, \Delta_{D}$ and $\Delta=\left(\sum_{j=1}^{D} \Delta_{j}^{2}\right)^{1 / 2}$. The interpolated value at a given location $\mathbf{x}$ is obtained using

$$
Z(\mathbf{x})=\Lambda(\mathbf{x}) * \Omega(\mathbf{x})
$$

where $*$ stands for convolution, $\Lambda(\mathbf{x})$ is the convolution kernel, and $\Omega(\mathbf{x})=\sum_{i} \delta\left(\mathbf{x}-\mathbf{x}_{i}\right) Z_{i}, \delta(\mathbf{x})$ being the Dirac- $\delta$ function. This equation can be written as

$$
Z(\mathbf{x})=\sum_{i} \Lambda\left(\mathbf{x}-\mathbf{x}_{i}\right) Z_{i}
$$

where $\sum_{i} \Lambda\left(\mathbf{x}-\mathbf{x}_{i}\right)=1, \forall \mathbf{x}$, showing that the value of $Z$ at $\mathbf{x}$ is estimated using the observations placed at $\mathbf{x}_{i}(i=1, \ldots, N)$.

The estimation of a quantity at locations different to those where data are acquired should only be performed when the spatial correlation is considered [14]. When spatial correlation is not taken into account, estimations at different locations are independent processes and the values at $\mathbf{x}_{i}$ should not be used to estimate the value of the image at $\mathbf{x}$. Kriging is a technique that explicitly considers the spatial correlation to estimate a quantity with spatial dependence along with its uncertainty [16]-[18]. It is a family of best linear unbiased estimators in the minimal squared sense. It is widely used in geostatistics and other experimental sciences such as geology, mining, biology, medicine, etc., where few data, placed irregularly, and with strong random fluctuations are available. Kriging has also been applied to image processing [19]-[21]. It has been shown that it leads to better results than adaptive Wiener filter [22].

To perform the interpolation, kriging considers the spatial correlation of the quantity by means of the variogram, which is defined as [17]

$$
2 \gamma(h)=\frac{1}{|N(\mathbf{h})|} \sum_{N(h)}\left(Z_{i}-Z_{j}\right)^{2}
$$


where $Z_{i}$ and $Z_{j}$ are the experimental data values at $\mathbf{x}_{i}$ and $\mathbf{x}_{j}$, respectively, the sum is over $N(\mathbf{h}) \equiv\left\{(i, j): \mathbf{x}_{i}-\mathbf{x}_{j}=\mathbf{h}\right\}$ and $|N(h)|$ is the number of distinct elements of $N(h)$. For simplicity, we assume that the spatial correlation is isotropic so that the spatial correlation only depends on the distance between locations.

In its most general form, kriging equations usually involve the inversion of large matrices, which can be quite time consuming [23]. When the locations of the measuring devices are uniformly located, as in the case of standard CCD cameras, kriging equations simplify and the interpolation can be obtained as a convolution [21], [24]. However, the uncertainty estimation still needs to be solved by the conventional matrix approach.

In Section II, we compare kriging with the standard statistical technique for determining the uncertainty given by (1). Since kriging considers the spatial correlation, the uncertainty decreases not only at the locations where observations are acquired, but also at their surroundings. Considering this, we propose a technique to compute the uncertainty of magnitudes with spatial dependence. The equation is similar to (1). However, the number of measurements $N$ is replaced by a function, $N_{\mathrm{EQ}}(\mathrm{x})$, that we have called the equivalent number of observations. $N_{\mathrm{EQ}}(\mathrm{x})$ function can be interpreted as the number of measurements that should be acquired at a given location, without considering the spatial correlation, to decrease the uncertainty as kriging does. We propose that $N_{\mathrm{EQ}}(\mathrm{x})$ is obtained as a convolution between a function assigned to each observation, that is related to the spatial correlation of the measured magnitude, and a function that represents the location of the observations. This procedure for uncertainty estimation is much faster than the standard kriging technique. In Section III, we perform some numerical simulations in order to analyze if the proposed technique produces similar results to those obtained with kriging. Finally, in Section IV, we apply this techique to one-dimensional (1-D) and two-dimensional (2-D) experimental images obtained with CCD cameras.

\section{UNCERTAINTY AS A CONVOLUTION}

The uncertainty estimated by kriging at the location where data are measured is not coincident, in its standard form [16]-[18], with that obtained using (1). For this reason, a modification of the kriging equations has been recently proposed in order to make both approaches compatible [24]. Using this modification of the kriging equations, we will analyze how kriging calculates the uncertainty for a case where the variogram is known, and only one observation is acquired $(N=1)$ at $\mathbf{x}_{1}$. In this case, the uncertainty can be easily computed [24], leading to

$$
\sigma_{K}^{2}(\mathbf{x})=I^{2}+2 \gamma\left(\left\|\mathbf{x}-\mathbf{x}_{1}\right\|\right)-s^{2}
$$

where $\sigma_{K}$ means that the uncertainty has been obtained with standard kriging, $s^{2}$ is the variance, and $I$ is the resolution of the measuring devices as defined in (1). In [25], it is shown that $\gamma(0)=s^{2}$ and then the uncertainty at $\mathbf{x}=\mathbf{x}_{1}$ is $\sigma_{K}\left(\mathbf{x}_{1}\right)=$ $\sqrt{I^{2}+s^{2}}$, which agrees with the uncertainty estimated using

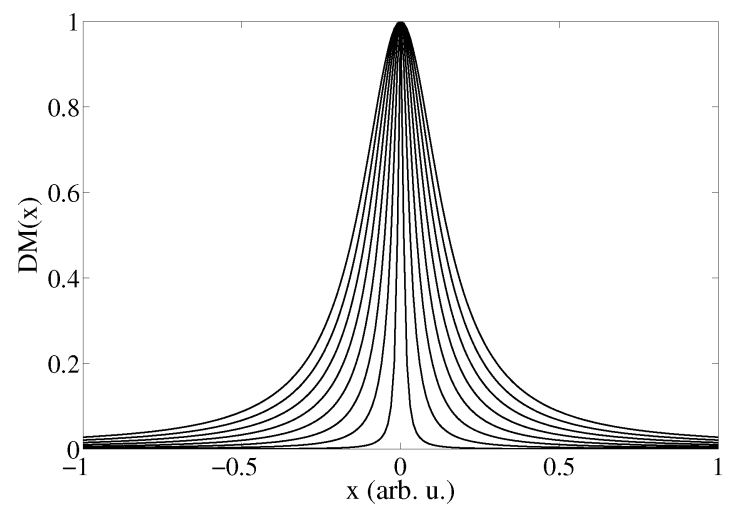

(a)

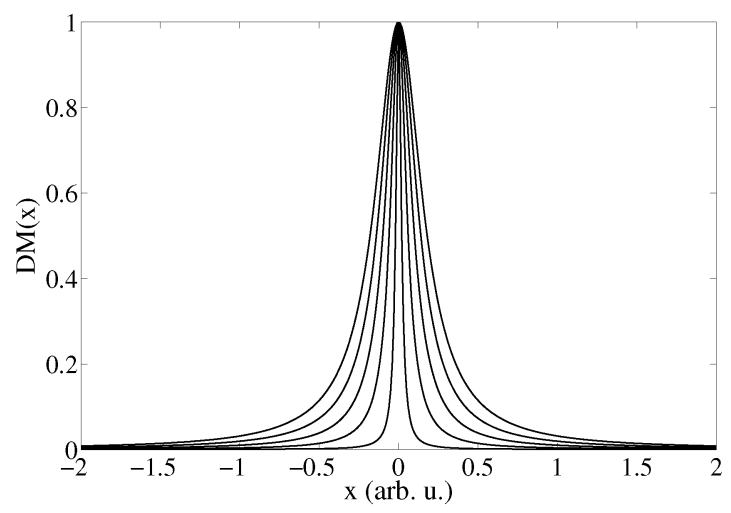

(b)

Fig. 1. (a) DM functions for $B=1, A=5$, and several values of $s$ $(0.05,0.15, \ldots, 0.75)$. For large values of $s$, the DM function widens. (b) DM functions for $s=0.2, A=5$, and several values of $B(0.5,1.5, \ldots, 4.5)$. For large values of $B$, the DM function widens.

(1). Also, when $N$ data are obtained at the same location $\mathbf{x}_{1}$, the uncertainty estimated by using kriging is

$$
\sigma_{K}^{2}(\mathbf{x})=I^{2}+\frac{s^{2}}{N}+2\left[\gamma\left(\left\|\mathbf{x}-\mathbf{x}_{1}\right\|\right)-s^{2}\right]
$$

which is also coincident at $\mathbf{x}=\mathbf{x}_{1}$ with (1). Comparing both approaches, we propose that the uncertainty estimated with kriging should be written as

$$
\sigma_{K}^{2}(\mathbf{x})=I^{2}+\frac{s^{2}}{N_{\mathrm{EQ}}(\mathbf{x})}
$$

where $N_{\mathrm{EQ}}(\mathrm{x})$ is a function that we shall call the equivalent number of observations. This equation can be inverted to calculate the value of $N_{\mathrm{EQ}}(\mathbf{x})$ from the uncertainty estimated by kriging

$$
N_{\mathrm{EQ}}(\mathbf{x})=\frac{s^{2}}{\sigma_{K}^{2}(\mathbf{x})-I^{2}} .
$$

$N_{\mathrm{EQ}}(x)$ is positive since $\sigma_{K}$ is greater than $I$. We can interpret $N_{\mathrm{EQ}}(x)$ as the number of observations that should be performed at a given location, without considering the spatial dependence, 


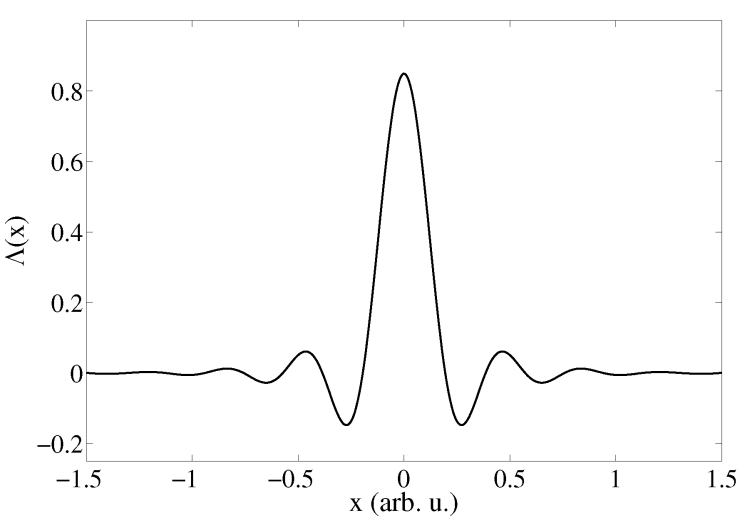

(a)

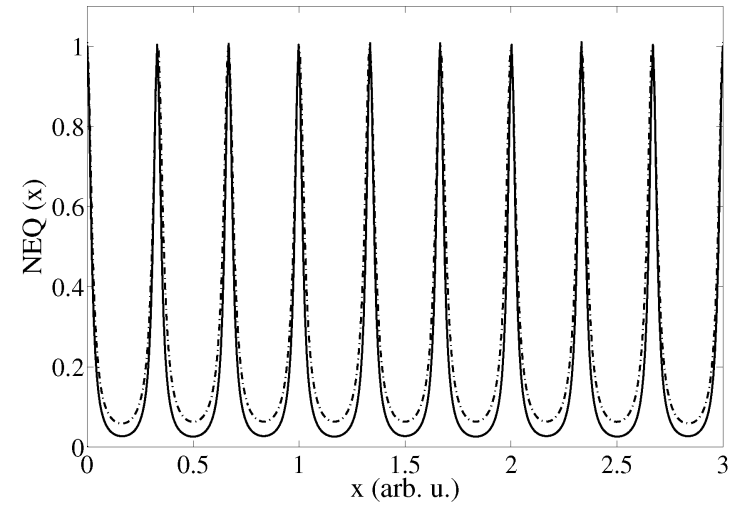

(c)

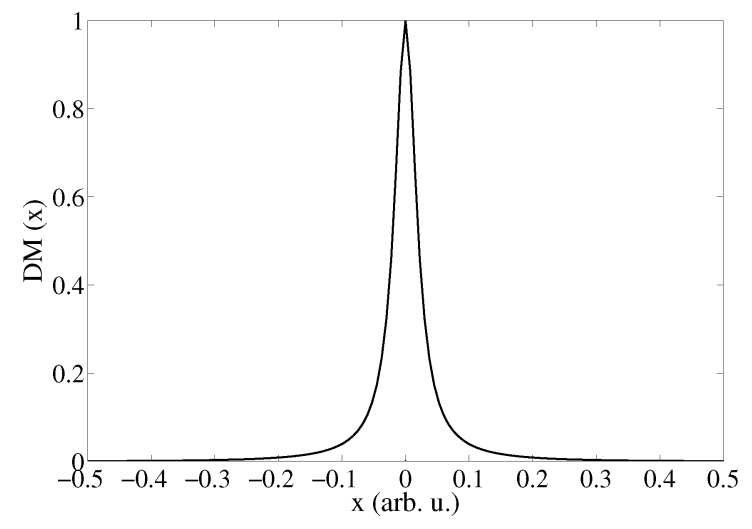

(b)

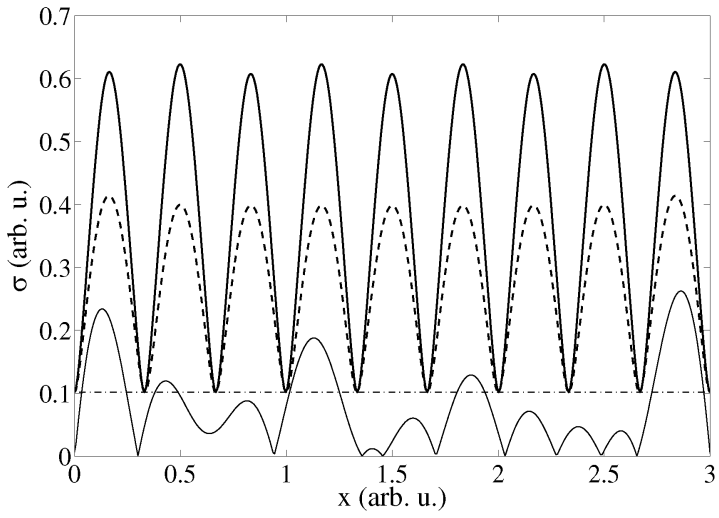

(d)

Fig. 2. Simulation of a sinusoidal function $f(x)=\sin (2 \pi x)$ with a random fluctuation of $s=0.02$ arb. $\mathbf{u}$, and a resolution of the measuring devices of $I=0.1$ arb. w. The sampling frequency is $v=4$ arb. $\mathrm{u}^{-1}$. (a) $\Lambda(x)$ function used for kriging estimation. (b) DM $(x)$ function. (c) $N_{\mathrm{EQ}}(x)$ calculated using kriging, (8) -dashed-, and as a convolution, (11) -solid-. Its value is approximately 1 at the location of the measuring devices, and it decreases strongly at the interstitials. (d) Uncertainty $\sigma(x)$ computed with standard kriging - dashed -, as a convolution using (12) -solid-, and $|Z(x)-\sin (2 \pi x)|$-thin-. The estimated uncertainty is approximately $\sqrt{s^{2}+I^{2}}$ at the location where data are placed and it is quite larger for locations between observations.

to decrease the uncertainty as kriging does. When only one observation is acquired at $\mathbf{x}_{1}$, from (5) and (8), $N_{\mathrm{EQ}}(x)$ becomes

$$
\operatorname{DM}(h)=:\left.N_{\mathrm{EQ}}(h)\right|_{N=1}=\frac{s^{2}}{2 \gamma(h)-s^{2}}
$$

where we have considered that $h=\left\|\mathbf{x}-\mathbf{x}_{1}\right\|$. We will refer to this function as the distributed measurement (DM). Since $\gamma(0)=s^{2}$, DM can be written exclusively in terms of the variogram function

$$
\operatorname{DM}(h)=\frac{\gamma(0)}{2 \gamma(h)-\gamma(0)} .
$$

$\operatorname{DM}(h)$ presents a maximum at $h=0, \operatorname{DM}(0)=1$, and it decreases when increasing $h$, provided that the spatial correlation decreases with the distance.

When observations are uniformly placed, we assume that $N_{\mathrm{EQ}}$ can be obtained from DM as a linear process in such a way that $N_{\mathrm{EQ}}$ can be obtained as the sum of individual DM functions assigned to each observation and centered at the location $\mathrm{x}_{i}$ where they are obtained

$$
N_{\mathrm{EQ}}(\mathbf{x})=\mathrm{DM}(\mathbf{x}) * \amalg(\mathbf{x})
$$

where $\amalg(\mathbf{x})=\sum_{i} \delta\left(\mathbf{x}-\mathbf{x}_{i}\right)$ is a function that characterizes the locations of the measuring devices. As a result, the uncertainty at each spatial location $\mathbf{x}$ can be obtained via

$$
\sigma_{C}^{2}(\mathbf{x})=I^{2}+\frac{s^{2}}{\operatorname{DM}(\mathbf{x}) * \amalg(\mathbf{x})}
$$

where $\sigma_{C}$ means that the uncertainty has been obtained as a convolution. This equation is a great simplification to the uncertainty estimation since, once the variogram is known, the uncertainty can be obtained as a convolution without having to calculate any inverse of a matrix. As a result, the time for computing the uncertainty using (12) is much shorter than following the standard approach [16], [17].

\section{A. Properties of DM}

As (9) and (12) show, the variogram is of major importance for determining the uncertainty of the interpolation when kriging method is applied. Normally, the experimental variogram obtained with (4) is not directly used, but it is fitted to a parametric function [17], [18]. Not all functions are valid, but the variogram must fit some mathematical properties [16], [17].

The way kriging performs the interpolation and the uncertainty estimation is quite sensitive to the functional shape of the variogram [18]. For example, when the variogram near the 


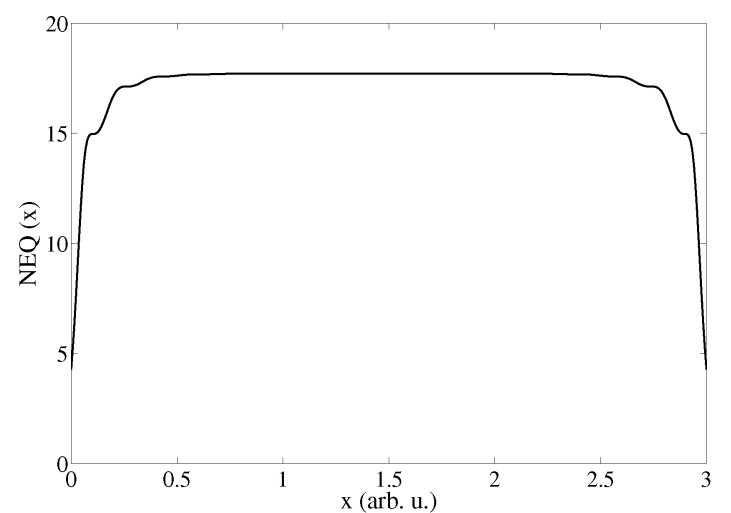

(a)

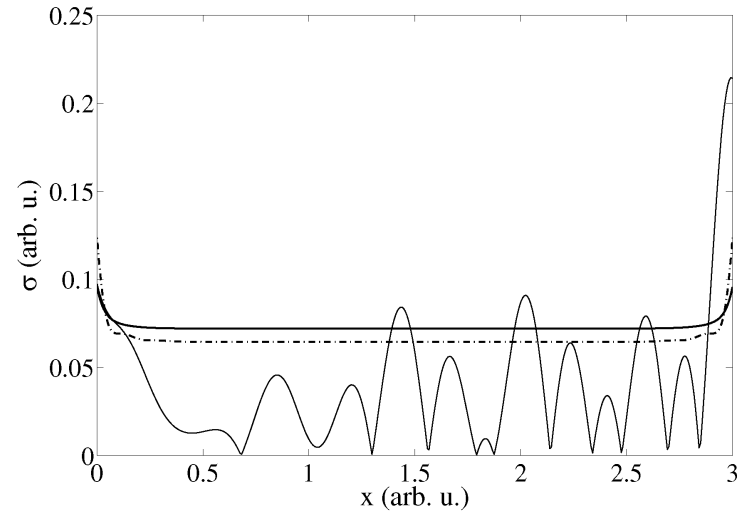

(b)

Fig. 3. Same case as Fig. 2, but now the sampling frequency is much larger $\left(v=100\right.$ arb. $\mathbf{u}^{-1}, s=0.4$ arb. $\mathbf{u} ., I=0.05$ arb u.). (a) $N_{\mathrm{EQ}}(x)$ function. (b) Uncertainty $\sigma(x)$ computed with standard kriging - dashed -, as a convolution using (12) -solid-, and $|Z(x)-\sin (2 \pi x)|$-thin-. Now, the estimated uncertainty $\sigma_{C}(x)$ is approximately constant.

origin presents a parabolic behaviour, the interpolated spatial quantity is differentiable (as is normally required). When the variogram is linear at the origin, the interpolated spatial quantity is continuous, but it is no longer differentiable. As an example, we will analyze the properties of DM for the case of the Gaussian variogram, since this is one of the most common cases

$$
\gamma(h)=s^{2}+A^{2}\left\{1-\exp \left[-\frac{1}{2}\left(\frac{h}{B}\right)^{2}\right]\right\}
$$

where $s$ is the standard deviation of the noise as shown in [25], $A$ is related to the maximum variability of the measured magnitude, and $B$ is the correlation length. To fit the experimental variogram to this parametric function, we have assumed that the spatial correlation is a local phenomenon. Then, we have not used the data of the experimental variogram from the first maximum on. They have been rejected in the fitting since it would indicate an increase of the correlation with the distance, such as in periodic signals.

Now, we will analyze how $\operatorname{DM}(h)$ behaves in terms of the different parameters involved. Introducing (13) into (9), we obtain

$$
\operatorname{DM}(h)=\frac{s^{2}}{s^{2}+2 A^{2}\left\{1-\exp \left[-\frac{1}{2}\left(\frac{h}{B}\right)^{2}\right]\right\}}
$$

which does not depend on the resolution of the measuring device $I$. In Fig. 1, we show $\mathrm{DM}(h)$ for several values of $s$ and $B$.

$\operatorname{DM}(h)$ does not vanish at infinity. Instead, $\lim _{h \rightarrow \infty} \operatorname{DM}(h)=s^{2} /\left(s^{2}+2 A^{2}\right)$. This means that the measured quantity does not increase arbitrarily when $h \rightarrow \infty$. Since DM does not vanish at infinity, it is not possible to calculate its width. However, the width of $\overline{\mathrm{DM}}(h)=\operatorname{DM}(h)-\lim _{h \rightarrow \infty} \operatorname{DM}(h)$ can be properly determined, leading to

$$
w=\sqrt{\int h^{2} \overline{\mathrm{DM}}(h) d h}=B \sqrt{\frac{L i_{3 / 2}\left(\frac{2 A^{2}}{2 A^{2}+s^{2}}\right)}{L i_{1 / 2}\left(\frac{2 A^{2}}{2 A^{2}+s^{2}}\right)}}
$$

where $L i_{n}(x)=\sum_{k=1}^{\infty} x^{k} / k^{n}$ is the polylogarithm function. It is clear that $w$ is directly proportional to the correlation length $B$. This proportionality has also been found in other parametric variograms.

The uncertainty $\sigma$ computed with (12) strongly depends on the ratio between the correlation length $B$ and the sampling distance $\Delta$. When $\Delta \ll B$, the $\operatorname{DM}(h)$ functions associated with each pixel overlap and then the uncertainty decreases at all locations. As a limiting case, when $B$ is infinite, then $\lim _{B \rightarrow \infty} \operatorname{DM}(h)=1$, and

$$
\sigma_{C}^{2}(\mathbf{x})=I^{2}+\frac{s^{2}}{N}
$$

$N$ being the total number of observations. In this case, the location of the observations is irrelevant and the uncertainty is equal to that obtained when all observations are acquired at the same place.

On the other hand, when $\Delta \gg B, N_{\mathrm{EQ}}(h)$ only presents large values at the neighborhoods of the observations, while it is approximately 0 at the rest of locations. As a limiting case, when the correlation length vanishes $(B=0)$, we get

$$
\mathrm{DM}(h)=\frac{s^{2}}{s^{2}+2 A^{2}}+\frac{2 A^{2}}{s^{2}+2 A^{2}} \sum_{i} \delta\left(\mathbf{x}-\mathbf{x}_{i}\right)
$$

and the uncertainty of the estimation is

$$
\sigma_{C}^{2}(\mathbf{x})=\left\{\begin{array}{ll}
I^{2}+s^{2}, & \mathbf{x}=\mathbf{x}_{i} \\
I^{2}+\frac{s^{2}+2 A^{2}}{N}, & \text { elsewhere }
\end{array} .\right.
$$

This means that observations are isolated. They make the uncertainty decrease only at the locations where they are acquired. At other locations the uncertainty is much greater (although not infinite, since the maximum variability of the magnitude $A$ is finite).

When the proposed theoretical variogram in (13) does not fit the experimental variogram, then the functional dependence of DM will be different to that of (14).

\section{NUMERICAL SimULATIONS}

In order to check that (12) is a valid approach for calculating the uncertainty of magnitudes with spatial dependence, we have simulated a 1-D band limited signal with a sinusoidal spatial 


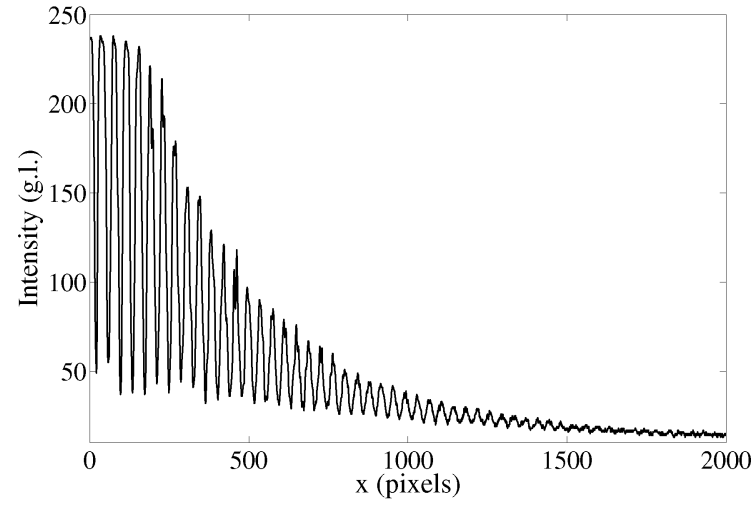

(a)

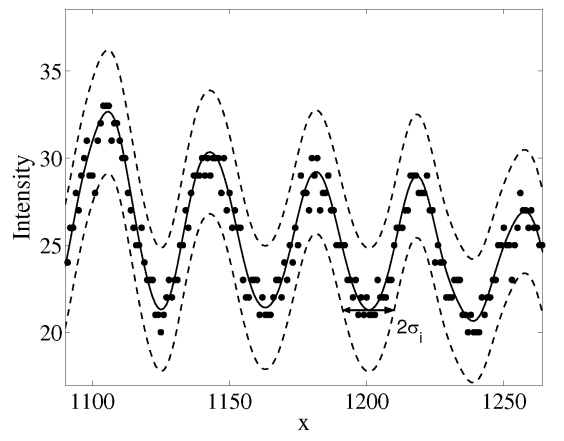

(c)

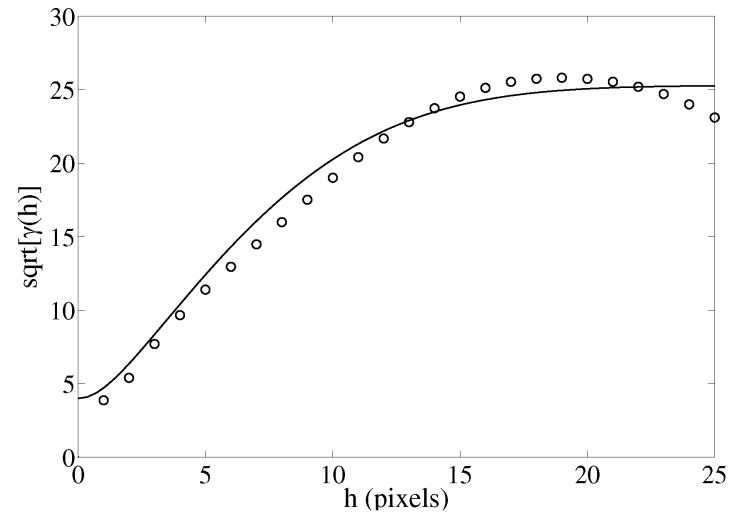

(b)

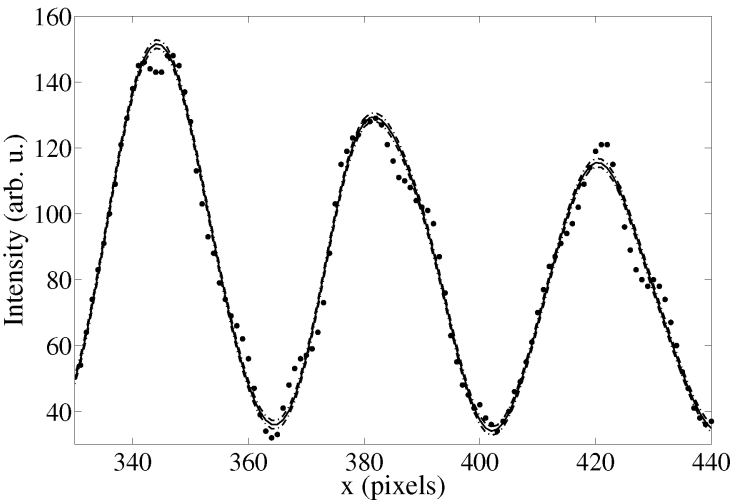

(d)

Fig. 4. (a) Far-field diffraction pattern for a steel wire with a diameter of $400 \mu \mathrm{m}$. (b) Experimental variogram - circles- and fit to Gaussian variogram, (13). (c) and (d) Zoom of Fig. 4(a) showing low and high diffraction orders, respectively. In these two figures, the estimation with kriging -solid- and the error bands, $Z(\varphi) \pm \sigma_{C}(\varphi)$, -dashed- are also shown, as well as the width of the diffraction minima.

dependence, $f(x)=\sin (2 \pi x)$, to which we have added two random processes

$$
Z_{i}=f\left(x_{i}\right)+p_{1}+p_{2}
$$

where $p_{1}$ is a zero-mean additive Gaussian probability distribution with standard deviation $s$ representing the random fluctuations of the measured quantity and $p_{2}$ is an additive uniform probability distribution between $[-k, k]$ representing the resolution of the measuring devices $I=k / \sqrt{3}$ [14]. The reason for this choice is that any band limited function can be described as a combination of sine functions. We have also tried with other functions, leading to equivalent results.

In a first example, we have simulated a case where $s=0.02$ arb. u, (arb. u stands for "arbitrary units"), $I=0.1$ arb. $\mathrm{u}$, and a sampling frequency $v=4$ arb. $\mathrm{u}^{-1}$. We have not performed an infinite sampling, but data are placed inside $x \in(0,3)$. We have calculated the variogram and, in Fig. 2(a) and (b), we have plotted the functions $\Lambda(x)$ and $\operatorname{DM}(x)$ obtained for this case. In Fig. 2(c), we have plotted the $N_{\mathrm{EQ}}(x)$ function using (8) and (11). At the location of the measuring devices $N_{\mathrm{EQ}}(x)$ is approximately 1 and it decreases strongly at the interstitials. As a consequence, the uncertainty is approximately $\sqrt{s^{2}+I^{2}}$ at the location where data are placed and it is quite larger for locations between observation [Fig. 2(d)].
On the other hand, when the sampling frequency increases, ( $v=100$ arb. u. ${ }^{-1}, s=0.4$ arb. u., $I=0.05$ arb. u). The situation is quite different. In such a case, $N_{\mathrm{EQ}}(x)$ is approximately constant and much greater than 1 [Fig. 3(a)]. As a consequence, the uncertainty is much smaller than $\sqrt{s^{2}+I^{2}}$ [Fig. 3(b)]. At the borders, the uncertainty increases due to finite sampling.

In both situations, the uncertainty estimated using (12) is quite similar to that obtained with kriging [24].

\section{Application to Image Processing}

In this section, we show two examples where the proposed technique for uncertainty estimation is applied to 1-D and 2-D images obtained with CCD cameras.

\section{A. One-Dimensional Images: Diffractometry}

Optical diffractometry is the most common technique for determining the dimensional parameters of small objects, such as spheres, slits, cylinders, and other simple objects [26]. This technique consists of illuminating the object with a monochromatic collimated light beam (normally a laser or a laser diode). The size of the object is determined from the far-field diffraction minima [27], [28]. As an example, the far-field diffraction pattern of a steel metallic wire with a nominal diameter of $400 \mu \mathrm{m}$ is shown in Fig. 4(a). This diffraction pattern is not smooth due to experimental imperfections, such as small defects in the cylinder surface, different detectability of photosensors, electronic noise, etc. The uncertainty estimation at the location of the diffraction minima is required to determine the uncertainty 


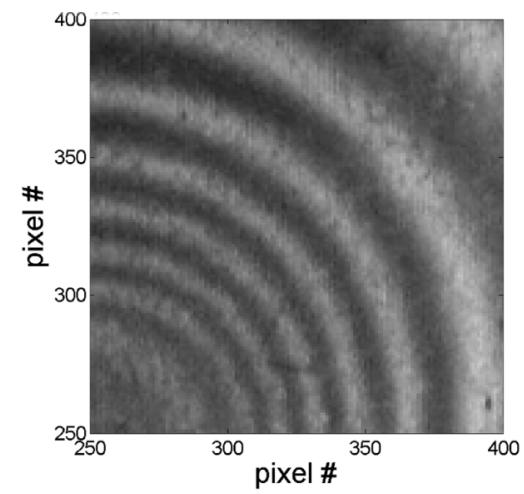

(a)

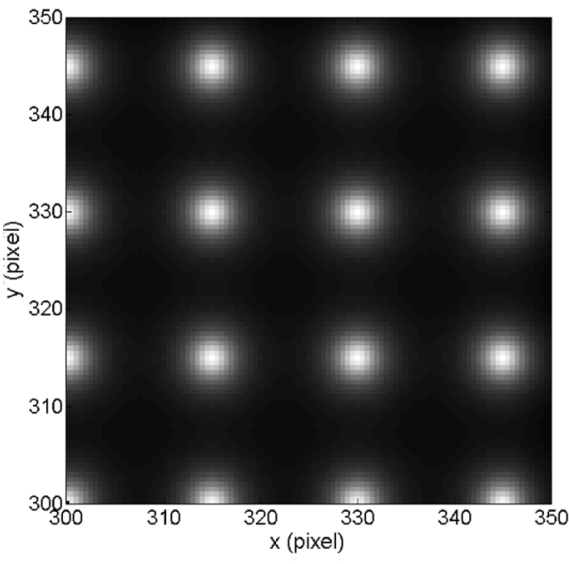

(c)

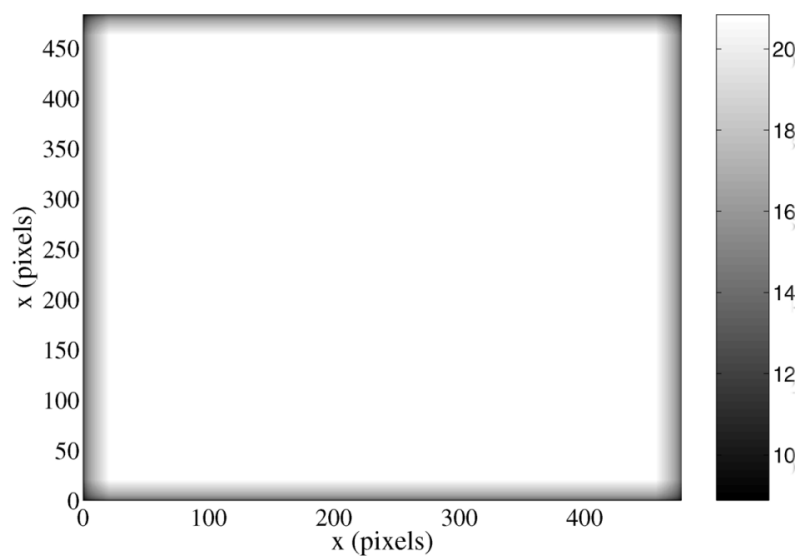

(b)

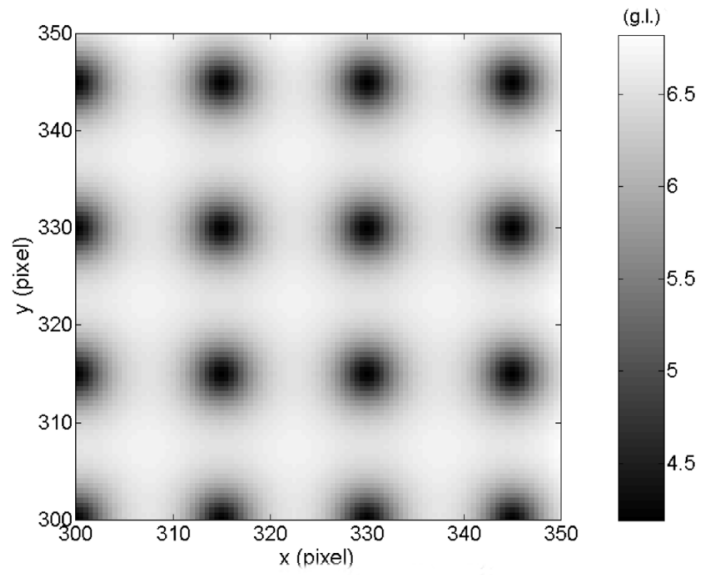

(d)

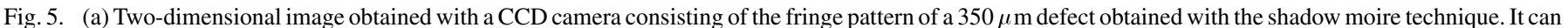

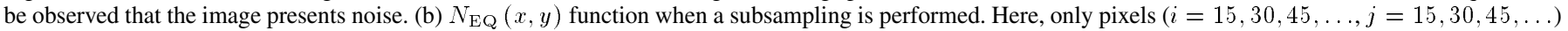
have been considered for the computation. (c) Uncertainty estimation $\sigma_{C}$ obtained using $N_{\mathrm{EQ}}(x, y)$. In this case, the uncertainty fluctuations are much larger.

of the dimensional parameters and also to experimentally validate the theoretical models used [20].

To determine the uncertainty by using the proposed technique, we have computed the variogram using 400 points fitting the increasing part of the variogram to a Gaussian variogram, (13), which is shown in Fig. 4(b). The fitting parameters obtained are $s=1.93$ g.l. (g.l. stands for :gray levels"), $A=24.96$ g.l., $B=7.06$ pixels. Using these parameters, we have calculated $\Lambda(x)$, as shown in [24], and DM $(x)$. With them, we have computed the filtered version of the diffraction pattern and the error bands. Fig. 4(c) and (d) shows two zones of such estimation along with the uncertainty estimation obtained with (12) and the experimental data. Due to the different contrast of the peaks, the widths of the diffraction minima vary. For the lower diffraction orders the contrast of the fringes is large, and the width of the minima is around 1.5 pixels. However, for high diffraction orders, the $S / N$ coefficient decreases, and the width of the minima is around 5 pixels. As a consequence, low-order diffraction minima are preferred for determining the dimensional parameters of the measured object.

\section{B. Two-Dimensional Images: Shadow Moire}

The proposed technique for uncertainty estimation based on convolution can also be applied to 2-D images since convolution can be performed using the fast Fourier transform. As an example, we have used a 2-D image of a fringe pattern that corresponds to a $350-\mu \mathrm{m}$ defect on a metallic plate using a shadow moire technique [Fig. 5(a)] [29]. This sample has been used in [24] for computing $\Lambda(x, y)$ using the kriging technique. The variogram at the origin is $\gamma(0)=24.0 \mathrm{~g} . \mathrm{l}^{2}$ and therefore the standard deviation is $s=\sqrt{\gamma(0)}=4.9$ g.l., the camera resolution being $I=1$ g.l. Uncertainty estimation using conventional kriging equations can be very time consuming since, for this case, the number of pixels was $512 \times 512$ so that kriging requires to compute the inverses of $262144 \times 262144$ matrices. As a consequence, only 1-D profiles were obtained. With the technique proposed in (12), the uncertainty using the $N_{\mathrm{EQ}}(x, y)$ function can be easily evaluated for the whole image. The result is that $N_{\mathrm{EQ}}(x, y) \approx 20.68$ is approximately constant except at the edges, where it decreases. As a consequence, the uncertainty is approximately constant, $\sigma_{C}(x, y) \approx 1.47 \mathrm{~g} .1$. This result is lower than that computed in [24] $\left(\sigma_{C} \approx 3.23\right.$ g.l. $)$ since, in that case, only $1-\mathrm{D}$ profiles were used, neglecting the contribution to $N_{\mathrm{EQ}}$ of the rest of the neighbouring pixels.

When the sampling distance is much larger than the correlation length of the variogram, $N_{\mathrm{EQ}}(x, y)$ is not constant, and the estimation of the uncertainty depends on the position. As an example, a subsampling of Fig. 5(a) is performed using only 1 of every 15 pixels. Then, $N_{\mathrm{EQ}}(x, y)$ is not constant presenting significant values only at the locations of the maintained pixels 
[Fig. 5(b)]. At the rest of locations, $N_{\mathrm{EQ}}$ decreases considerably, and the error in the estimations increases [Fig. 5(c)].

\section{CONCLUSIONS}

The kriging technique can be applied to perform the interpolation of images obtained with CCD cameras. In addition, the uncertainty of the estimation can also be determined with kriging, which is important when the image is used for metrological purposes. However, the uncertainty estimation needs to be solved by the conventional matrix approach. In this work, we have compared kriging with the standard statistical technique for determining the uncertainty and we have proposed a new procedure for estimating the uncertainty of images which reduces the computing time considerably. Uncertainty is computed using an equation similar to the standard technique of uncertainty estimation. However, the number of measurements $N$ is replaced by a function that we have called equivalent number of observations $N_{\mathrm{EQ}}(\mathbf{x})$. The $N_{\mathrm{EQ}}(\mathbf{x})$ function is obtained as a convolution between a function assigned to each observation, that exclusively depends on the variogram, and a function that represents the location of the observations. The proposed technique for uncertainty estimation has been applied to numerical simulations and to 1-D and 2-D images obtained with CCD cameras. The results are in agreement with those obtained with kriging.

\section{ACKNOWLEDGMENT}

The authors thank Dr. J. Zoido, Dr. J. A. Quiroga, Dr. J. Alda, and Dr. A. Luis for their help and fruitful discussions.

\section{REFERENCES}

[1] C. E. Shannon, "Communication in presence of noise," Proc. IRE, vol. 37, pp. 20-21, 1949.

[2] K. F. Cheung and R. J. Marks, II, "Imaging sampling below the Nyquist density without aliasing," J. Opt. Soc. Amer. A, vol. 7, no. 1, pp. 92-105, 1990.

[3] H. P. Urbach, "Generalised sampling theorem for band-limited functions," Math. Comput. Model., vol. 38, pp. 133-140, 2003.

[4] A. Stern and B. Javidi, "Sampling in the light of Wigner distribution," J. Opt. Soc. Amer. A, vol. 21, no. 3, pp. 360-366, 2004.

[5] _ - "Analysis of practical sampling and reconstruction from Fresnel fields," Opt. Eng., vol. 43, no. 1, pp. 239-250, 2004.

[6] G. C. Holst, CCD Arrays, Cameras and Displays. Bellingham, WA: Society for Photo-Optical Instrumentation Engineers, 1996.

[7] J. M. López-Alonso, J. Alda, and E. Bernabeu, "Principal components characterization of noise for infrared images," Appl. Opt., vol. 41, pp. 320-331, 2002.

[8] J. M. López-Alonso and J. Alda, "Matricial representation of the transfer function for aliased systems," Proc. SPIE, vol. 4719, pp. 208-219, 2002.

[9] A. J. Jerri, "The shannon sampling theorem - Its various extensions and applications," Proc. IEEE, vol. 65, pp. 1565-1596, Nov. 1977.

[10] W. K. Pratt, Digital Image Processing. New York: Wiley, 1978.

[11] M. Pawlak and U. Stadmüller, "Recovering band-limited signals under noise," IEEE Trans. Inf. Theory, vol. 42, no. 5, pp. 1425-1438, May 1996.

[12] M. Unser, "Sampling - 50 years after Shannon," Proc. IEEE, vol. 88, pp. 569-587, Apr. 2000.
[13] P. Bevington, Data Reduction and Error Analysis for the Physical Sciences. New York: McGraw-Hill, 1969.

[14] Guide to the Expression of the Uncertainty in Measurement. Geneva: International Standardisation Organisation (ISO), 1995.

[15] M. Tabib-Azar, , W. Göpel, J. Hesse, and J. N. Zemel, Eds., "Sensor parameters," in Sensors, Fundamental and General Aspects. Weinheim, Germany: VCH, 1989, vol. 1.

[16] R. Christiensen, Linear Models for Multivariate, Time Series, and Spatial Data. Berlin, Germany: Springer-Verlag, 1985.

[17] N. Cressie, Statistics for Spatial Data. New York: Wiley, 1991.

[18] J. P. Chilès and P. Delfiner, Geostatistics: Modeling Spatial Uncertainty. New York: Wiley, 1999.

[19] D. Mainy, J. P. Nectoux, and D. Renard, "New developments in data processing of noisy images," Mater. Characteriz., vol. 36, pp. 327-334, 1996.

[20] E. Bernabeu, I. Serroukh, and L. M. Sanchez-Brea, "A geometrical model for wire optical diffraction selected by experimental statistical analysis," Opt. Eng., vol. 38, no. 8, pp. 1319-1325, 1999.

[21] W. Y. V. Leung, P. J. Bones, and R. G. Lane, "Statistical interpolation of sampled images," Opt. Eng., vol. 40, no. 4, pp. 547-553, 2001.

[22] T. D. Pham and M. Wagner, "Image enhancement by kriging and fuzzy sets," Int. J. Pattern Recognit., vol. 14, no. 8, pp. 1025-1038, 2000.

[23] W. H. Press, S. A. Teukolski, W. T. Vetterling, and B. P. Flannery, Numerical Recipes in C. New York: Cambridge Univ. Press, 1992.

[24] L. M. Sanchez-Brea, "Determination of the optimum sampling frequency of noisy images by spatial statistics," Appl. Opt., vol. 44, no. 16 , pp. 3276-3283, 2005.

[25] L. M. Sanchez-Brea and E. Bernabeu, "On the standard deviation in charge-coupled device cameras: A variogram-based technique for nonuniform images," J. Electron. Imag., vol. 11, no. 2, pp. 121-126, 2002.

[26] M. Born and E. Wolf, Principles of Optics, 6th ed. Oxford, U.K.: Pergamon, 1980.

[27] J. F. Fardeau, "New laser sensors for wire diameter measurement," Wire J. Intern., vol. 22, no. 1, pp. 42-51, 1989.

[28] T. K. Millard and T. A. Herchenreder, "Automatic diameter measurement: State of the art," Wire J. Intern., vol. 24, no. 12, pp. 61-69, 1991.

[29] G. Cloud, Optical Methods of Engineering Analysis. Cambridge, U.K.: Cambridge Univ. Press, 1998.

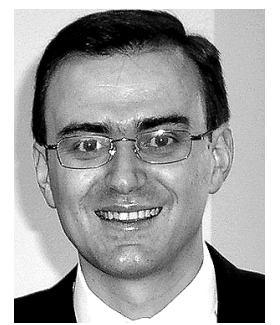

Luis Miguel Sanchez-Brea received the M.Sci. degree in 1996 and the Ph.D. degree in physics in 2001 from the Universidad Complutense de Madrid, Madrid, Spain.

He has been with the Universidad Complutense de Madrid as an Assistant Professor, with Fagor Automation S. Coop. as a Research and Development Technician, and is currently a "Ramon y Cajal" Researcher at the Optics Department, Universidad Complutense de Madrid. He has written around 25 papers in his fields of research, which are applied optics metrology and image processing.

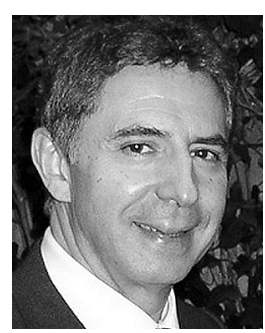

Eusebio Bernabeu received the M.Sci. degree in 1966 and the Ph.D. degree in 1969 in physics from the University of Zaragoza, Zaragoza, Spain.

Since 1986, he has been a Professor with the Physics Faculty, Universidad Complutense de Madrid, Madrid, Spain. He has been with L'Ecole Normale Superieur, Laboratoire de l'Horloge Atomique, Paris, France, the International Centre of Theoretical Physics of Trieste, and at the Universities of Zaragoza, Autonoma de Barcelona, and the Complutense de Madrid. His current principal areas of interest are optoelectronics, optical sensors, fiber-optical devices, and optical industrial inspection and metrology. He has written more than 250 papers and ten books.

Dr. Bernabeu is a member of OSA, SPIE, and EPS. 